\title{
TU/e EmonOWEN

\section{Data-driven controller unfalsification with analytic update applied to a motion system}

\section{Citation for published version (APA):}

Helvoort, van, J. J. M., Jager, de, A. G., \& Steinbuch, M. (2008). Data-driven controller unfalsification with analytic update applied to a motion system. IEEE Transactions on Control Systems Technology, 16(6), 12071217. https://doi.org/10.1109/TCST.2008.919429

DOI:

10.1109/TCST.2008.919429

Document status and date:

Published: 01/01/2008

\section{Document Version:}

Publisher's PDF, also known as Version of Record (includes final page, issue and volume numbers)

\section{Please check the document version of this publication:}

- A submitted manuscript is the version of the article upon submission and before peer-review. There can be important differences between the submitted version and the official published version of record. People interested in the research are advised to contact the author for the final version of the publication, or visit the $\mathrm{DOI}$ to the publisher's website.

- The final author version and the galley proof are versions of the publication after peer review.

- The final published version features the final layout of the paper including the volume, issue and page numbers.

Link to publication

\section{General rights}

Copyright and moral rights for the publications made accessible in the public portal are retained by the authors and/or other copyright owners and it is a condition of accessing publications that users recognise and abide by the legal requirements associated with these rights.

- Users may download and print one copy of any publication from the public portal for the purpose of private study or research.

- You may not further distribute the material or use it for any profit-making activity or commercial gain

- You may freely distribute the URL identifying the publication in the public portal.

If the publication is distributed under the terms of Article 25fa of the Dutch Copyright Act, indicated by the "Taverne" license above, please follow below link for the End User Agreement:

www.tue.nl/taverne

Take down policy

If you believe that this document breaches copyright please contact us at:

openaccess@tue.nl

providing details and we will investigate your claim. 


\title{
Data-Driven Controller Unfalsification With Analytic Update Applied to a Motion System
}

\author{
Jeroen van Helvoort, Bram de Jager, and Maarten Steinbuch
}

\begin{abstract}
Ellipsoidal unfalsified control is a plant-model-free, data-driven control design method. It recursively checks, using available data, whether the ability of a controller to meet a predefined performance requirement is (un)falsified. The set of unfalsified controllers is described by an ellipsoid in the control parameter space. The update of the ellipsoid employing new measurements can be computed analytically, hence, it is computationally cheap. This adaptive scheme is applied to an experimental motion system, namely to an industrial inkjet printer at a sample rate of $1 \mathrm{kHz}$. The results clearly show that the algorithm updates the control parameter set when the performance requirement is not met with the currently implemented one. The resulting closed-loop behavior resembles the predefined reference model in the dominant frequency range.
\end{abstract}

Index Terms-Adaptive control, control engineering, ellipsoids, motion control, plant-model-free, unfalsified control.

\section{INTRODUCTION}

\section{A. Data-Driven Control}

D ATA-DRIVEN control design methods focus on finding controllers, using merely input-output data of the system. Since these methods do not require a plant and/or disturbance model in the design of the controller, optimistic modeling assumptions and conservative model-error bounds are omitted. Nevertheless, if a model or other plant information or assumptions are available, they can be used in the initialization of the algorithm. In fact, its use is encouraged, for instance, in the design of the controller structure, in the selection of the initial controller, and in the determination of the performance requirements to ensure that all information about the plant is used in the design of the controller such that the chances that a stabilizing and performing controller results are maximized.

There are numerous motives to support data-driven control design, e.g., a priori definition of controller complexity, adaptation to specific disturbances, elimination of model mismatch/assumptions. Furthermore, sometimes the time or means lack to accurately model the system at hand for control, including the execution of identification experiments and model reduction. With data-driven control design methods, the model is non-crucial, hence plant/disturbance modeling might even be omitted.

Manuscript received May 21, 2007; revised December 3, 2007. Manuscript received in final form January 10, 2008. First published June 10, 2008; current version published October 22, 2008. Recommended by Associate Editor C. Knospe.

The authors are with the Technische Universiteit Eindhoven, 5600 MB Eindhoven, The Netherlands (e-mail: j.j.m.v.helvoort@ieee.org; a.g.de.jager@tue.nl; m.steinbuch@tue.nl).

Color versions of one or more of the figures in this paper are available online at http://ieeexplore.ieee.org.

Digital Object Identifier 10.1109/TCST.2008.919429

\section{B. Unfalsified Control}

An emerging data-driven control design method is unfalsified control [11]. This data-driven, plant-model-free control design approach recursively falsifies controllers that fail to satisfy a performance requirement, given measured data. The controllers whose ability to meet the performance requirement is not contradicted by the available data are said to be unfalsified, and together they form the unfalsified set. If the unfalsification procedure is repeated over time, a recursive algorithm is constructed.

Several approaches exist to implement an unfalsified control algorithm. In early works, a discrete set of controllers was selected, such that only a finite set of controllers was to be evaluated. As a consequence, the computational load was directly linked to the number of initial candidate controllers. In later works, a specified control law was regarded in combination with continuously distributed control parameter sets, hence, with infinitely many controllers.

In [14], the unfalsification algorithm is applied only after a batch of data is collected, batch-wise adaptation. As a benefit, the computational load to compute the unfalsified set is irrelevant, since no hard constraint is put on the calculation time if the time between two batches is not strict.

In [13], the unfalsification algorithm is implemented at each sample instance, which we will refer to as continuous adaptation. This approach puts a hard constraint on the computational load, since the calculation of the unfalsified set and the selection of an unfalsified controller from this set has to be finished within one sample time. However, with the current implementation all past measurement data is considered such that the size of the problem grows over time. To address this issue, the past measurement data can be discarded, as is done in the simulation example periodically every $0.5 \mathrm{~s}$, or a recursive implementation should be adopted. But even then, both this method and the aforementioned method involve a linear programming optimization problem. This property makes them less suitable for real-time implementation with continuous adaptation on a system with a fast sample rate, such as a motion system, since the optimization might be too computationally demanding to finish within every sample time even for relatively simple control laws.

An optimization problem can be avoided, if an approximation of the unfalsified set is considered. In [2], the unfalsified set is approximated by an outer-bounding ellipsoid, the update of which is computed by the ellipsoid algorithm [1]. This algorithm provides an analytical update, which is computationally cheap. However, the update algorithm uses one half-plane through the center of the ellipsoid, the "cutting plane," as an approximation of the separation between control parameter sets 
that are falsified by the new measurement data and control parameter sets that are unfalsified by it. As a consequence, this cutting plane can only be applied when the current control parameter set (which in [2] is the center of the current ellipsoidal unfalsified set) is falsified. When the current control parameter set is unfalsified, the ellipsoid is not changed and the newly acquired unfalsification information is discarded. Other algorithms can be used, to tighter bound the approximation of the intersection of the ellipsoid with the region unfalsified by the new measurement data, which typically also is an ellipsoid, however, these often result in optimization problems, which we want to avoid.

\section{Ellipsoidal Unfalsified Control}

The optimal (i.e., minimum-volume) outer-bounding ellipsoidal approximation of the intersection of two ellipsoids can be computed analytically only in a few specific cases. The solutions known are when the ellipsoid is exactly sliced in half (as is employed in [2]), when the centers of two intersecting ellipsoids coincide, or when at least one ellipsoid degenerates into two parallel half-spaces [7]-[10].

This last case is employed in ellipsoidal unfalsified control (EUC) [6]. Here, by construction, the separation between control parameter sets that are falsified by new measurement data and control parameter sets that are unfalsified, is defined by two parallel half-spaces. Consequently, the approximation of the intersection can be computed analytically without the need for the current control parameter set to be falsified, and moreover, two cutting planes are defined, neither of which is restricted to pass through the center of the ellipsoid.

In [6], sufficient conditions are provided to guarantee the stability of this plant-model-free control design method, based on the results in [12]. It only needs the fundamental feasibility assumption, i.e., the assumption that in the initial candidate controller pool there is at least some region with control parameter sets that fulfill the performance requirement at all times. If this assumption is fulfilled, stability can be guaranteed. Since they are only sufficient, a stable adaptive control system might still result even if these conditions and assumptions are not met.

Recently, in [4] and [5], we were able to extend the algorithm to multiple-input-multiple-output (MIMO) systems. However, here we report on the experimental results with a single-input-single-output (SISO) motion system.

\section{Additional Value of This Work}

Clearly, the strength of a data-driven control design method is best proven with real-life experiments. While simulations might provide useful insights on isolated phenomena, the method typically is desired to work with the entire ensemble of phenomena as encountered in experiments, such as friction, sensor and actuator noise, saturations, quantization, computational delays, to mention only a few. Besides, contrary to simulations where the computation time at each sampling instant is unrestricted because real-time evaluation is not required, with real-time implementation on a physical system the sample time imposes a limit on the computation time.
Experience has shown that applicability in simulations is not a sufficient condition for applicability in experiments. The performance of many algorithms that work well in simulations deteriorates drastically when implemented in experiments. The assessment of the causes where this discrepancy originates, e.g., from certain neglected phenomena, inconsistent simulation design, or inherent assumptions, is left to the reader.

In this paper, the results of the application of this data-driven control design method to a motion system are shown and evaluated, together with the realization of the specific design choices. The theory of EUC is slightly extended compared to previous work, to serve the need of the experimental environment. Namely, the controller structure is extended with a nonlinear term in $r$ to compensate for Coulomb friction and the bound on the performance requirement is relaxed where high performance is not relevant. The main contribution of this paper, therefore, is contained in the argued design of and application in an experiment, rather then in the development of the theory of EUC. For the latter, the reader is referred to [6].

\section{E. Structure of This Work}

We start with an introduction to EUC in Section II. The experimental setup is introduced in Section III and the controller design is illustrated in Section IV. The results and evaluation are provided in Sections V and VI, respectively. Section VII contains the conclusion.

\section{ELLIPSOIDAL UNFALSIFIED CONTROL}

In [6], the EUC approach is introduced. This section shows the approach of this data-driven, plant-model-free controller design method.

\section{A. Controller Unfalsification}

Definition 1 (Controller Unfalsification): A controller is said to be falsified by measurement information if this information is sufficient to deduce that the performance requirement would be violated if that controller were in the feedback loop. Otherwise, the controller is said to be unfalsified [11].

Key issue in unfalsified control is the efficient deduction of falsification, which is achieved by application of the fictitious reference signal (see Section II-D). Besides, the set of unfalsified controllers should be updated efficiently if controllers are falsified. This is especially an issue if a continuous set of controllers is regarded, hence, with infinitely many controllers. A continuous set of controllers is realized by imposing an interval on the values of the parameters in a specified shared control law, in contrast to selecting a finite set of predefined candidate controllers. The exact description of the region of unfalsified controllers might be too complex for fast computation and, furthermore, the complexity grows in time. Therefore, an approximation is made of the region. In EUC, the region of unfalsified parameters is approximated by an ellipsoid. Furthermore, new measurement data defines two parallel half-spaces of unfalsified controllers, the optimal intersection with an ellipsoid can be computed analytically. Here, optimality means that the approximation is outer-bounding, to not incorrectly falsify controllers, and of minimal volume. The analytic computation enables a fast algorithm, since no explicit optimization has to be performed. 
In the remainder of this section the design choices of EUC are presented.

\section{B. Ellipsoidal Unfalsified Set}

Consider the definition of the true unfalsified set.

Definition: The true unfalsified set $E_{\text {tus }}$ is the set of controllers that are currently unfalsified by all available measurement data.

In EUC, the true unfalsified set is approximated by an ellipsoid. This ellipsoid is used as a representation for the true unfalsified set and will be denoted as the unfalsified set $\mathcal{E}$.

The unfalsified set at time $t_{k-1}$ is described by

$$
\mathcal{E}\left(t_{k-1}\right)=\left\{\theta \mid\left(\theta-\theta_{c}\left(t_{k-1}\right)\right)^{T} \Sigma^{-1}\left(t_{k-1}\right) \cdot\left(\theta-\theta_{c}\left(t_{k-1}\right)\right) \leq 1\right\}
$$

with $\theta \in \mathbb{R}^{p}$ the control parameter set, $\theta_{c}\left(t_{k-1}\right) \in \mathbb{R}^{p}$ the center of the ellipsoid, and $\Sigma\left(t_{k-1}\right) \in \mathbb{R}^{p \times p}$ the symmetric, positive definite matrix that describes the shape and size of the ellipsoid.

\section{Performance Requirement}

A key issue in unfalsified control is the performance requirement. The ability of controllers to meet the predefined performance requirement directly determines whether a controller is unfalsified or not, see [11].

In EUC, the performance requirement is defined as a (timedependent) bound $0<\Delta\left(t_{k}\right)<\infty$ on the tracking error plus a $\kappa$-weighted control effort, $\kappa\left(t_{k}\right)>0$

$$
\left|W(q) e\left(t_{k}\right)\right|+\kappa\left(t_{k}\right)\left|u\left(t_{k}\right)\right| \leq \Delta\left(t_{k}\right)
$$

with

$$
e\left(t_{k}\right)=G_{m}(q) r\left(t_{k}\right)-y\left(t_{k}\right)
$$

Here, $q$ is the backward time-shift operator (i.e., $q * t_{k}=t_{k-1}$ ), $W(q)$ is a stable filter, e.g., a low-pass filter to reduce the effect of outliers and $G_{m}(q)$ is the desired closed loop dynamics.

The $\ell_{\infty}$ performance requirement (2) guarantees that destabilizing behavior of a candidate controller results in its falsification. This property is needed for the guarantee of a stable adaptive control system, see Section II-A and [4] and [6].

To perform the check (2) for all controllers would be a tedious job, all the more since it has to be done for all possible operating conditions. Therefore, the fictitious reference is considered which enables evaluation of controllers that are not implemented.

\section{Fictitious Reference}

The fictitious reference is a fictitious signal, used to evaluate the performance of a controller. For a given controller, the fictitious reference is defined as the reconstructed reference signal that would have resulted in exactly the measured input and output of the plant if that controller would have been implemented. A schematic representation of the construction of the fictitious reference is shown in Fig. 1. Measurement data

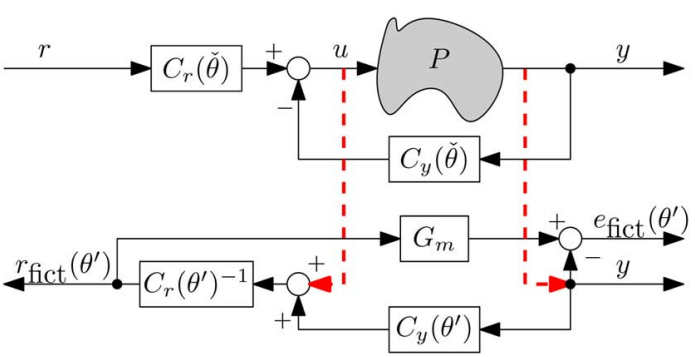

Fig. 1. Schematic representation of the construction of the fictitious reference $r_{\text {fict }}$ and fictitious error $e_{\text {fict }}$.

$[u, y]$ is acquired with controllers $C_{r}(\check{\theta})$ and $C_{y}(\check{\theta})$ in the feedback loop. Then $r_{\text {fict }}\left(\theta^{\prime}\right)$ is constructed, such that had $r_{\text {fict }}\left(\theta^{\prime}\right)$ been applied to the system with controllers $C_{r}\left(\theta^{\prime}\right)$ and $C_{y}\left(\theta^{\prime}\right)$ this would exactly have resulted in the measured $[u, y]$. For the setup of Fig. 1, the fictitious reference is given by

$$
r_{\text {fict }}\left(\theta^{\prime}\right)=C_{r}\left(\theta^{\prime}\right)^{-1}\left(u+C_{y}\left(\theta^{\prime}\right) y\right) .
$$

The concept of a fictitious reference enables the evaluation of controllers, even if they were not in the loop at the time of the measurement, for the fictitious error (7) instead of the actual tracking error (3) can be used to evaluate (2). This substitution enables the construction of a region containing the controllers that are unfalsified by current measurement data at time $t_{k}$

$$
\begin{aligned}
\mathcal{U}\left(t_{k}\right) & =\left\{\theta|| W(q) e_{\text {fict }}\left(\theta, t_{k}\right)\left|+\kappa\left(t_{k}\right)\right| u\left(t_{k}\right) \mid \leq \Delta\left(t_{k}\right)\right\} \\
& =\left\{\theta \mid-\hat{\Delta}\left(t_{k}\right) \leq W(q) e_{\text {fict }}\left(\theta, t_{k}\right) \leq \hat{\Delta}\left(t_{k}\right)\right\}
\end{aligned}
$$

with

$$
\begin{aligned}
e_{\text {fict }}\left(\theta, t_{k}\right) & =G_{m}(q) r_{\text {fict }}\left(\theta, t_{k}\right)-y\left(t_{k}\right) \\
\hat{\Delta}\left(t_{k}\right) & =\Delta\left(t_{k}\right)-\kappa\left(t_{k}\right)\left|u\left(t_{k}\right)\right| .
\end{aligned}
$$

It should be noted that $\mathcal{U}\left(t_{k}\right)$ is empty for $\hat{\Delta}\left(t_{k}\right)<0$.

\section{E. Controller Structure}

The controller structure is chosen such that the fictitious reference generator, $r_{\text {fict }}\left(\theta, t_{k}\right)$, is affine in the control parameter set $\theta$. Furthermore, $r_{\text {fict }}\left(\theta, t_{k}\right)$ depends on $u\left(t_{k}\right), y\left(t_{k}\right)$ and filtered versions thereof and also possibly on nonlinear functions of $u\left(t_{k-1}\right), y\left(t_{k}\right)$ and past values thereof. Then, a general form for the fictitious reference generator is given by

$$
\begin{aligned}
r_{\text {fict }}\left(\theta, t_{k}\right) & =\left[\begin{array}{c}
u\left(t_{k}\right) \\
\Lambda_{u}(q) \otimes u\left(t_{k-1}\right) \\
\Lambda_{y}(q) \otimes y\left(t_{k}\right) \\
f\left(u\left(t_{k-1}\right), y\left(t_{k}\right), q\right)
\end{array}\right]^{T}\left[\begin{array}{c}
\theta_{1} \\
\underline{\theta}_{2} \\
\underline{\theta}_{3} \\
\underline{\theta}_{4}
\end{array}\right] \\
& =w\left(u\left(t_{k}\right), y\left(t_{k}\right), q\right)^{T} \theta
\end{aligned}
$$

where $\Lambda_{u}(q)$ and $\Lambda_{y}(q)$ are vectors of asymptotically stable linear filters, $\otimes$ denotes the Kronecker product, and $f\left(u\left(t_{k-1}\right), y\left(t_{k}\right), q\right)$ is a vector with nonlinear convergent 


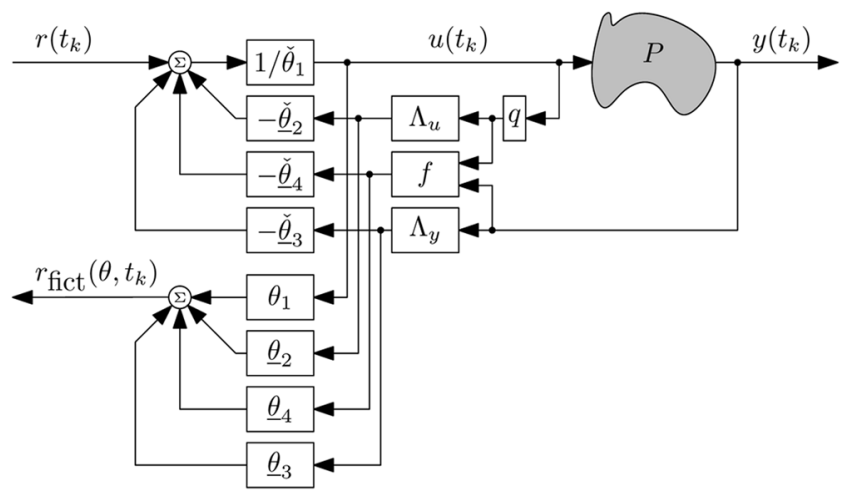

Fig. 2. Shows a schematic representation of controller (11) and fictitious reference generator (9).

functions that are bounded in amplitude for all $u\left(t_{k-1}\right)$ and $y\left(t_{k}\right)$. Clearly, (10) defines the set of candidate controllers, as it follows that

$$
u\left(t_{k}\right)=\left[\begin{array}{c}
r\left(t_{k}\right) \\
\Lambda_{u}(q) \otimes u\left(t_{k-1}\right) \\
\Lambda_{y}(q) \otimes y\left(t_{k}\right) \\
f\left(u\left(t_{k-1}\right), y\left(t_{k}\right), q\right)
\end{array}\right]^{T}\left[\begin{array}{c}
1 / \check{\theta}_{1} \\
-\check{\ddot{\theta}}_{2} / \check{\theta}_{1} \\
-\breve{\theta}_{3} / \check{\theta}_{1} \\
-\underline{\check{\theta}}_{4} / \check{\theta}_{1}
\end{array}\right]
$$

Fig. 2 schematic representation of the controller (11) and fictitious reference generator (9).

The linear controller part of Fig. 2 equals the controller of Fig. 1 by substitution of $C_{r}(\check{\theta})=(1) /(\check{\theta})_{1}+\check{\theta}_{2}^{T} \Lambda_{u}(q) q$ and $C_{y}(\check{\theta})=\left(\check{\theta}_{3}^{T} \Lambda_{y}(q)\right) /\left(\check{\theta}_{1}+\underline{\theta}_{2}^{T} \Lambda_{u}(q) q\right)$.

Controller structure (9) imposes the constraint that $C_{r}(\check{\theta})$ and $C_{y}(\check{\theta})$ have a shared parametrization of the denominator, however, the parametrization itself is free to choose. Furthermore, the numerator of $C_{r}(\check{\theta})$ does not depend on $\check{\theta}$, but it is influenced by the selection of the denominators of the filters in $\Lambda_{u}(q)$.

The region $\mathcal{U}\left(t_{k}\right)$ (6) defines two parallel half-spaces for $\hat{\Delta}\left(t_{k}\right)>0$, as can be seen by substitution of (10) into (7)

$$
\mathcal{U}\left(t_{k}\right)=\left\{\theta \mid-1 \leq \phi_{k}^{T} \theta-y_{k} \leq 1\right\}
$$

with

$$
\begin{aligned}
\phi_{k} & =\frac{W(q) G_{m}(q) w\left(u\left(t_{k}\right), y\left(t_{k}\right), q\right)}{\hat{\Delta}\left(t_{k}\right)} \\
y_{k} & =\frac{W(q) y\left(t_{k}\right)}{\hat{\Delta}\left(t_{k}\right)} .
\end{aligned}
$$

\section{F. Noise Analysis}

EUC does not employ any model of the plant, therefore, no distinction can be made between plant output and noise. At most a distinction can be made between a reproducible part and a nonreproducible part of $y\left(t_{k}\right)$, part of which may originate from noise. The influence of the non-reproducible part of $y\left(t_{k}\right)$ (as of the reproducible part, obviously) on $e_{\text {fict }}\left(\theta, t_{k}\right)$ and $u\left(t_{k}\right)$ has to be bounded, otherwise, the controller is falsified.

\section{G. Update Ellipsoidal Unfalsified Set}

The unfalsification information for measurement data up to $t_{k-1}$ is represented by the ellipsoid $\mathcal{E}\left(t_{k-1}\right)(1)$. New measurement data provides new unfalsification information, which is represented by the two parallel half-spaces $\mathcal{U}\left(t_{k}\right)$ (12). The intersection $\mathcal{E}\left(t_{k-1}\right) \cap \mathcal{U}\left(t_{k}\right)$ defines the region of controllers, that are unfalsified both by past and by present measurement data. For preservation of the ellipsoidal shape of the unfalsified set, the intersection $\mathcal{E}\left(t_{k-1}\right) \cap \mathcal{U}\left(t_{k}\right)$ is approximated by a minimum-volume outer-bounding ellipsoid $\mathcal{E}\left(t_{k}\right)$, which is then used in the next time step to represent the unfalsification information for measurement data up to $t_{k}$.

Because $\mathcal{U}\left(t_{k}\right)$ defines two parallel half-spaces, an analytic solution exists to compute $\mathcal{E}\left(t_{k}\right)$, as was shown in [9]. In [6], this algorithm is described for EUC. It is shown that the update depends on the location of the parallel half-spaces with regard to the ellipsoid, and that the volume of $\mathcal{E}$ decreases monotonically.

To guarantee a limited number of distinctive ellipsoids, a minimum decrease in volume is imposed on the update algorithm. The limited number of distinctive ellipsoids is used to guarantee stability, see Section II-A.

\section{H. Controller Selection}

A controller that is unfalsified by the available unfalsification information is to be inserted in the loop. Or in other words, one controller inside $\mathcal{E}\left(t_{k-1}\right) \cap \mathcal{U}\left(t_{k}\right)$ is to be implemented.

Since all controllers in $\mathcal{E}\left(t_{k-1}\right) \cap \mathcal{U}\left(t_{k}\right)$ are unfalsified by the available information, the selection of the controller that is to be implemented basically is arbitrary. In [6], controller selection (13) is proposed. With this algorithm, a finite number of controller switches is guaranteed, and it is shown that the controller is in the strict intersection $\mathcal{U}\left(t_{m}\right) \cap \ldots \cap \mathcal{U}\left(t_{n}\right)$ if the ellipsoid is not changed in the interval $\left[t_{m}, t_{n}\right]$.

$$
\check{\theta}\left(t_{k}\right)= \begin{cases}\check{\theta}\left(t_{k-1}\right), & \text { if }\left|\gamma\left(t_{k}\right)\right| \leq 1 \\ \Gamma\left(t_{k}, \alpha\right) \check{\theta}\left(t_{k-1}\right)+ & \\ \left(1-\Gamma\left(t_{k}, \alpha\right)\right) \theta_{c}\left(t_{k}\right), & \text { if }\left|\gamma\left(t_{k}\right)\right|>1\end{cases}
$$

with $\check{\theta}\left(t_{k-1}\right)$ the currently implemented controller parameters, and

$$
\begin{aligned}
\Gamma\left(t_{k}, \alpha\right) & =\alpha \frac{\operatorname{sign}\left(\gamma\left(t_{k}\right)\right)-\gamma_{c}\left(t_{k}\right)}{\gamma\left(t_{k}\right)-\gamma_{c}\left(t_{k}\right)} \\
\alpha & \in\left[0, \epsilon_{\alpha}\right], \quad \epsilon_{\alpha}<1 \\
\gamma\left(t_{k}\right) & =\phi_{k}^{T} \check{\theta}\left(t_{k-1}\right)-y_{k} \\
\gamma_{c}\left(t_{k}\right) & =\phi_{k}^{T} \theta_{c}\left(t_{k}\right)-y_{k} .
\end{aligned}
$$

Note that for $\left|\gamma\left(t_{k}\right)\right|>1, \check{\theta}\left(t_{k-1}\right)$ is falsified by current measurement data [see (12)]. Parameter $\alpha$ determines the "aggressiveness" of the controller switch algorithm. By imposing $\alpha$ strictly smaller then 1 , a maximum number of controller switches per ellipsoids is guaranteed.

\section{Stability}

In [6], sufficient conditions are provided to guarantee stability of this data-driven adaptive control system. Only (sufficient) assumption is that there exists a region of controllers 
in the initial candidate controller set with controllers that satisfy the performance requirement at all times (feasibility assumption). The conditions to guarantee that the EUC system is bounded-input-bounded-output (BIBO) stable, see also [12], are summarized by:

1) adaptive control problem is feasible;

2) stably causally-left-invertible (SCLI) candidate controllers are considered, in combination with the $l_{\infty}$ performance specification (2);

3) maximum number of controller switches is limited.

The candidate controllers are SCLI for $\breve{\theta}_{1} \neq 0$. Condition 3 is satisfied, because the number of distinctive ellipsoids as well as the number of controller switches per ellipsoid are limited.

\section{J. Summary and Remarks}

Several design choices are explicitly specified for EUC, such that a fast evaluation of an infinite number of candidate controllers is feasible. First, the unfalsified set is specified by an ellipsoid that describes a continuous region of control parameter sets. Second, the performance requirement (2) is adopted, which, in combination with the fictitious reference generator (9) and controller structure (10), results in two parallel half-spaces. The intersection of the ellipsoid and the two parallel half-spaces is approximated by a minimum-volume outer-bounding ellipsoid, which can be computed analytically and which is used as the unfalsified set in the next time step. Accordingly, the same arithmetics can be used every time step.

An algorithm for the deterministic selection of the control parameter set to be implemented is given.

With the current algorithm, the control parameter set can be adapted even if the unfalsified set remains unchanged. Also the opposite is true, that is, the unfalsified set can be adapted while the control parameter set remains unchanged.

\section{EXPERIMENTAL SETUP}

In this section, the experimental setup is introduced. As in a model-based controller design procedure, some characteristics of the plant are identified (i.e., a frequency response estimate of the plant and an estimate of the Coulomb friction). These characteristics are used to design a preliminary controller and to evaluate the behavior of the plant with EUC. It should be noted that these characteristics are not directly used in the EUC algorithm, although in the design of $G_{m}(q), w(\cdot), \Delta\left(t_{k}\right), \kappa\left(t_{k}\right)$ and $r\left(t_{k}\right)$ some plant knowledge is helpful.

\section{A. Description Printer Setup}

Experiments have been performed on a motion system, to illustrate the effectiveness of the method. As a benchmark testbed, a stripped-down industrial inkjet printer is utilized, which is depicted in Fig. 3. The carriage for the printheads moves along a guidance rail and is driven by a dc-motor through a belt transmission. The position of the carriage is measured by a linear optical encoder, with an increment size of 4.26 $10^{-5} \mathrm{~m} / \mathrm{in}$. The system is sampled at $1 \mathrm{kHz}$.

1) Coulomb Friction: The first characteristic, observed when conducting experiments on the printer setup, is a high level of Coulomb friction. This parasitic effect is induced by the sleeve bearing between the carriage and the guidance rail. The effort

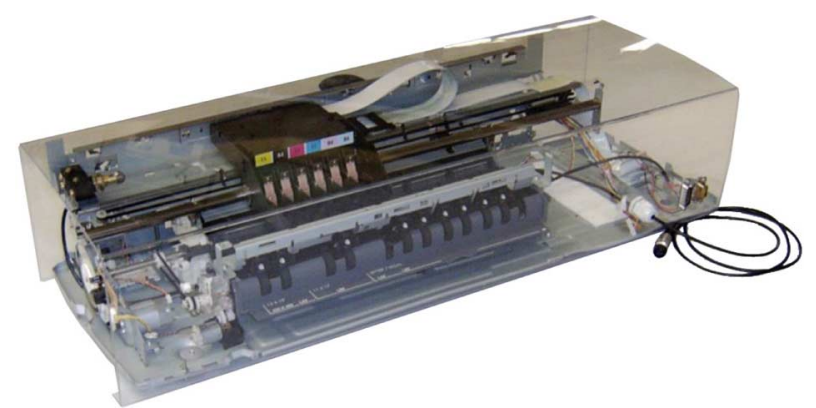

Fig. 3. Photo of stripped-down inkjet printer setup. The transparent cover is attached for safety.

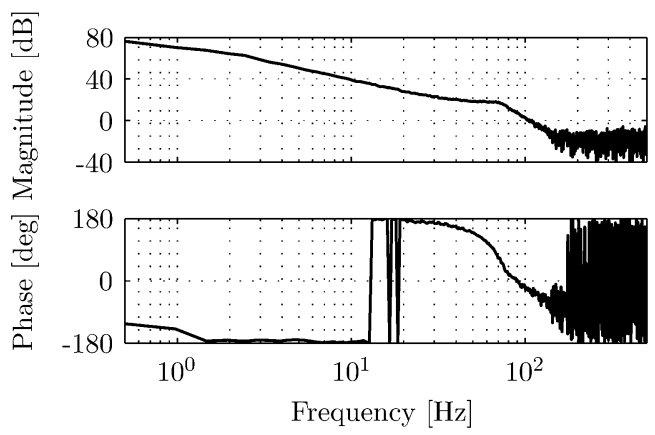

Fig. 4. Bode plot of the transfer of the input voltage of the motor to the position of the carriage for the experimental setup, as shown in Fig. 3.

consumed for this effect accounts for roughly $40 \%$ of the available input.

2) Frequency Response: To obtain an estimate of the frequency response function of the printer setup, a measurement is conducted of the sensitivity function $S=(1+C P)^{-1}$. This frequency response will not be used in the design of the controllers, but only to evaluate the controllers and to provide insight in the dynamics of the system at hand. For the sensitivity measurement, a rudimentary feedback controller is implemented. To limit the influence of friction, the level of Coulomb friction is estimated and roughly compensated with a feedforward signal. Moreover, a constant velocity trajectory is imposed, to assure that the carriage is not in the stick region. A pseudo-random signal is added to the controller output and the influence on the plant input is captured. This way, the sensitivity function $S=(1+C P)^{-1}$ is computed. Since $C$ is known (transfer of the implemented feedback controller), the only unknown is the plant $P$.

A Bode plot of the transfer of the input voltage of the amplifier of the dc-motor to the position of the carriage (in encoder increments), computed from the sensitivity measurement, is shown in Fig. 4. This transfer shows the characteristic low-frequent rigid body mode $(1) /\left(\left(M s^{2}\right)\right)$ of motion systems, with a phase shift due to time delay and a resonance at higher frequencies.

3) Remark on the Obtained Frequency Response: The frequency response, as obtained with the sensitivity measurement, is a good starting point for controller design. However, the frequency response function for similar setups might differ, a phenomenon that is not captured in this single measurement. To cover variations induced, e.g., by the production process or by wear, an uncertainty model might be included. The construction 
of such an uncertainty model is not a straightforward procedure and might end up in an overly conservative design. Therefore, we apply EUC to this setup, since it adapts to the actual system at hand and to the disturbances present during operation.

\section{EXPERIMENT DESIGN}

In Section III, the experimental setup is introduced. In this section, the choices for the EUC experiment are discussed.

\section{A. Goals}

Characteristic for an inkjet printer is the repetitive nature of the motion of the carriage. To deliver the ink to the paper, the carriage moves over the paper in multiple strokes. During a stroke, the carriage ideally moves with constant velocity, and the turn at the end of a stroke is as fast as possible. To accomplish a maximum region of constant velocity, a triangular wave is chosen as the trajectory $r\left(t_{k}\right)$ of the carriage. The amplitude is representative for printing of an A4/letter size page.

If the carriage is in the printing area, the performance requirement is strict. However, if the carriage is not in the printing area, the requirements with regard to accuracy are less strict. Therefore, different levels are chosen for the performance requirement $\Delta$. The relaxation of the performance requirement outside the printing area allows fast reversal of the carriage.

The desired closed-loop behavior $G_{m}(q)$ of the controlled system is prescribed by a second-order low-pass reference model, to impose low-frequent tracking and high-frequent noise suppression

$$
G_{m}(q)=\mathcal{Z}\left(\frac{\omega_{n}^{2}}{s^{2}+2 \zeta \omega_{n} s+\omega_{n}^{2}}\right)
$$

with

$$
\begin{aligned}
\omega_{n} & =100 \\
\zeta & =0.8 .
\end{aligned}
$$

Here, $\mathcal{Z}(\cdot)$ denotes the zeroth-order-hold transformation from the $s$-domain to the $q$-domain.

The triangular reference is given by

$$
r\left(t_{k}\right)=2 f A \int_{0}^{t_{k}} \operatorname{sign}(\sin (2 \pi f t)) d t
$$

with $f=0.75 \mathrm{~Hz}$ and $A=7050$ inc. and sampled at $1 \mathrm{kHz}$ with a zeroth-order-hold. The frequency content of $r\left(t_{k}\right)$ is shown in Fig. 5. The performance requirement is chosen to decay exponentially, and is 10 times larger if the carriage is not in the printing area

$$
\Delta\left(t_{k}\right)= \begin{cases}10+1000 e^{-0.1 t_{k}}, & \text { for } 500 \leq r\left(t_{k}\right) \leq 6550 \\ 10\left(10+1000 e^{-0.1 t_{k}}\right), & \text { otherwise }\end{cases}
$$

The initial value $\Delta(0)$ is determined from preliminary experiments, by investigation of the error with the initial controller.

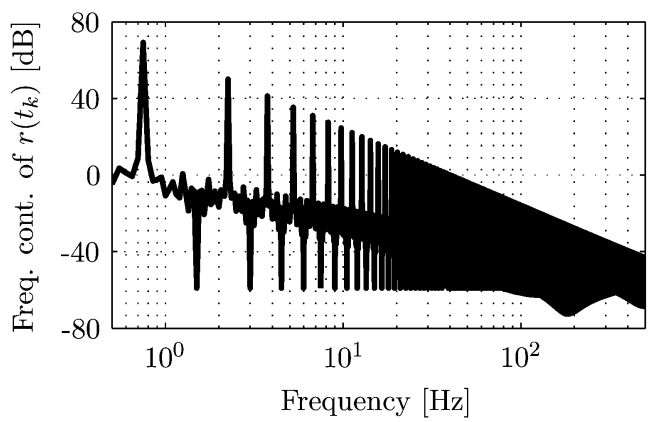

Fig. 5. Frequency content of triangular reference $r\left(t_{k}\right)$ (21). The magnitude of the peaks (at odd multiples of $0.75 \mathrm{~Hz}$ ) decreases for increasing frequency.

The final value $\Delta\left(t_{\infty}\right)$ is determined by repeatedly performing the experiment with diminishing $\Delta\left(t_{\infty}\right)$. The exponential decay is included to prescribe a gradually improving tracking performance and to allow for transient behavior.

The influence of $u\left(t_{k}\right)$ on the performance requirement is only marginally weighted, by setting its weighting factor to $\kappa\left(t_{k}\right)=10^{-3}$. The maximum observed value of $u\left(t_{k}\right)$ was 5.1935 .

The filter to reduce the effect of noise and outliers $W(q)(2)$ is set to 1 .

\section{B. Controller Structure}

The controller structure is chosen as a collection of low-pass and high-pass filters, and a nonlinear element of $r\left(t_{k}\right)$

$$
w\left(u\left(t_{k}\right), y\left(t_{k}\right), r\left(t_{k}\right), q\right)=\left[\begin{array}{c}
u\left(t_{k}\right) \\
\frac{q-0.25 q^{2}}{1-0.99 q} u\left(t_{k}\right) \\
\frac{q-0.99 q^{2}}{1-0.25 q} u\left(t_{k}\right) \\
q u\left(t_{k}\right) \\
y\left(t_{k}\right) \\
\frac{q-0.25 q^{2}}{1-0.99 q} y\left(t_{k}\right) \\
\frac{q-0.99 q^{2}}{1-0.25 q} y\left(t_{k}\right) \\
q y\left(t_{k}\right) \\
\operatorname{sign}\left((1-q) r\left(t_{k}\right)\right)
\end{array}\right] .
$$

The linear filters are chosen such that a lead-filter and a lag-filter are in the candidate controller set. Furthermore, symmetry between $u\left(t_{k}\right)$ and $y\left(t_{k}\right)$ is introduced. Typically, for motion systems a $(1) /\left(\left(M s^{2}\right)\right)$ trend is observed at low-frequencies, with some additional delay. This implies that a lead-lag filter suffices to result in a stable control system with sufficient margins. It is, therefore, expected that the combination of lead- and lag-filters results in a feasible adaptive control problem. The breakpoints of the filters are chosen well below and above the breakpoint of $G_{m}(q)$.

The nonlinear element represents the direction of motion and is included to compensate the high level of Coulomb friction, as encountered in the experimental setup. It is not included in the definition of $r_{\text {fict }}\left(\theta, t_{k}\right),(10)$, so it is regarded as an exogenous signal, which is plausible because it only depends on the direction of movement of $r\left(t_{k}\right)$. Nonetheless, it might introduce 
an offset in the fictitious reference, especially at the reversal of the carriage motion. Yet, the benefit that the carriage starts moving even with the initial controller, despite the high level of Coulomb friction, is eminent with this setup.

Note that the controller structure is not designed to achieve perfect tracking. Perfect tracking is not an issue here, merely tracking within the user defined bounds $\pm \Delta\left(t_{k}\right)$.

\section{Initial Parameters}

Several parameters need to be defined a priori, which will be discussed here.

As initial controller, a P-controller is constructed with a very small gain plus a unit compensation for the Coulomb friction

$$
\check{\theta}(0)=\left[\begin{array}{llllllllll}
1000 & 0 & 0 & 0 & 1 & 0 & 0 & 0 & -1000
\end{array}\right]^{T} .
$$

The resulting control effort is given by

$$
u\left(t_{k}\right)=\frac{1}{1000}\left(r\left(t_{k}\right)-y\left(t_{k}\right)\right)+\operatorname{sign}\left((1-q) r\left(t_{k}\right)\right) .
$$

The center $\theta_{c}(0)$ of the initial ellipsoidal unfalsified set is chosen identical to the initial candidate controller set $\check{\theta}(0)$. The initial ellipsoid is chosen as a sphere with radius 100

$$
\Sigma(0)=10^{4} \rrbracket_{9}
$$

The stepsize of the controller switching algorithm (15) is chosen $\alpha=0.9$, which is at some distance from the bounds of the unfalsified set. This choice enforces a moderate stepsize of the controller parameters, while still guaranteeing a finite number of controller switches. A smaller $\alpha$ might result in a faster convergence of the selected controller parameter set, however, it also induces larger transient behavior. A larger $\alpha$ results in smaller, but more frequent, controller switches. The value of $\alpha$ chosen here is a suitable compromise.

The maximum volume ratio is constrained by setting $\epsilon(\nu)=$ $0.99<1$, which results in the dismissal of updates of the ellipsoids for which the volume ratio is only marginally smaller then $1\left(\delta_{V}>0.99999\right)$ (see [6]). A smaller $\epsilon(\nu)$ results in a decreased limit of the number of distinctive ellipsoids at the expense of the dismissal of more possible updates of the ellipsoid.

\section{RESULTS}

In this section, the results of the experimental implementation of EUC are shown, and an evaluation of the results is provided. In the next section, an evaluation of the overall performance of EUC is given.

\section{A. Time Domain}

In Fig. 6, tracking error $G_{m}(q) r\left(t_{k}\right)-y\left(t_{k}\right)$ is shown as a function of time, together with bound $\pm \Delta\left(t_{k}\right)$. Since the performance bound $\Delta\left(t_{k}\right)$ switches between two values, see (22), two distinct levels are observed. Because the control effort is only

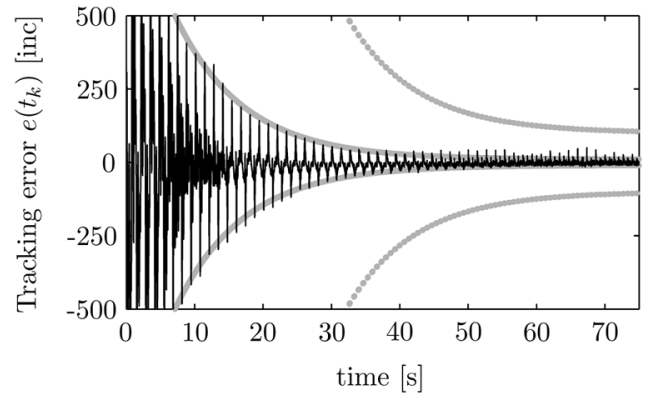

Fig. 6. Tracking error as a function of time (black line) and bounds $\pm \Delta\left(t_{k}\right)$ (grey dots). Due to the switching of the bound $\Delta\left(t_{k}\right)$, see (22), two distinctive levels are observed.

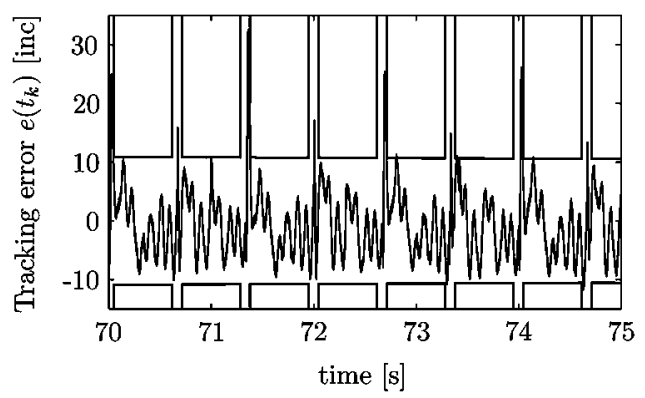

Fig. 7. Zoom of tracking error after $70 \mathrm{~s}$ (black line) and bounds $\pm \Delta\left(t_{k}\right)$ (grey lines). Peaks in the tracking error are observed during reversal of the carriage motion.

weighted marginally, the two signals shown in Fig. 6 give a good indication on the unfalsification of the currently implemented controller. Namely, if the tracking error is outside the bounds $\pm \Delta\left(t_{k}\right)$, the currently implemented controller is falsified.

The initial controller (25) results in a large tracking error. Every time that the currently implemented controller is falsified, the controller parameters are adapted. In Fig. 7, it is shown that the controller after $70 \mathrm{~s}$ satisfies the performance requirements at all these times. During reversal of the carriage motion, peaks in the tracking error are observed. However, due to relaxation of the performance requirements outside the printable area, these peaks are not restrictive.

The evolution of the controller parameters $\breve{\theta}\left(t_{k}\right)$ is shown in Fig. 8. Fig. 8 also shows the center of the ellipsoid $\theta_{c}\left(t_{k}\right)$, as well as the bounds of $\mathcal{E}\left(t_{k}\right)$ in the direction of the parameter $\theta_{c, i} \pm \Sigma_{i, i}^{-1 / 2}$. Because the currently implemented controller parameters are frequently falsified, see Fig. 6 , the parameters are also adapted many times.

Initially, the bounds for $\theta_{6}$ decrease rapidly, whereas simultaneously the bounds of the other parameters increase. This increase results from the outer bounding ellipsoidal approximation. Nonetheless, the volume of the unfalsified set decreases monotonically, as can be seen from Fig. 9, which shows the evolution of $\operatorname{det}\left(\Sigma\left(t_{k}\right)\right)$ over time. The combination of a simultaneous increase in some directions and decrease in others is also visible in the lobes on $\theta_{c, i} \pm \Sigma_{i, i}^{-1 / 2}$, which result from a changing orientation of $\mathcal{U}\left(t_{k}\right)$ over time, combined with the outer-bounding approximation. 


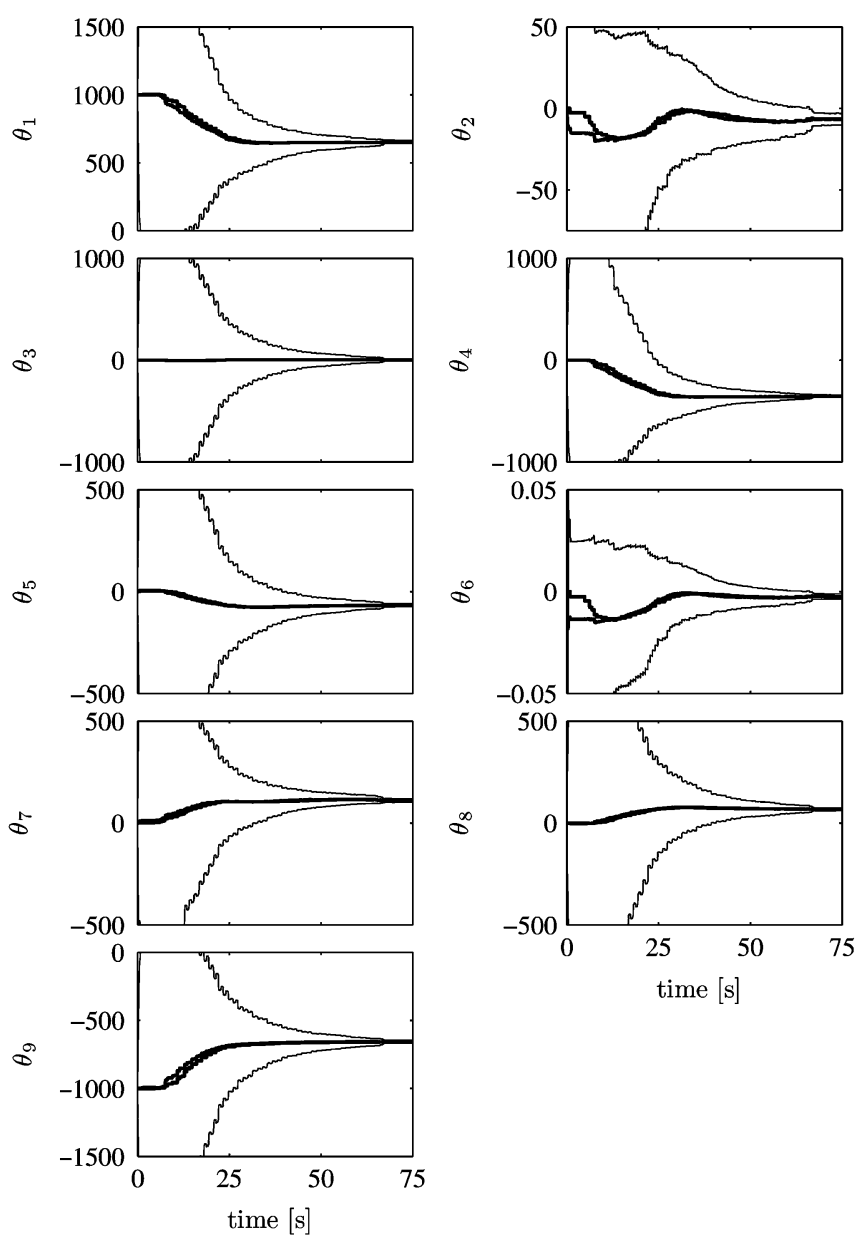

Fig. 8. Evolution of controller parameters $\check{\theta}\left(t_{k}\right)$ over time (black pronounced line). Also shown is the center of the ellipsoid $\theta_{c}\left(t_{k}\right)$ (gray) and the bounds $\theta_{c, i} \pm \Sigma_{i, i}^{-1 / 2}$.

The values of the controller parameters $\check{\theta}(75)$ and the center of the unfalsified set $\theta_{c}(75)$ are

$$
\check{\theta}(75)=\left[\begin{array}{c}
653.4618 \\
-6.5962 \\
0.8944 \\
-351.3421 \\
-67.4793 \\
-0.0024 \\
109.4123 \\
67.2029 \\
-656.9903
\end{array}\right] \quad \theta_{c}(75)=\left[\begin{array}{c}
653.3262 \\
-6.6972 \\
0.9075 \\
-351.4874 \\
-67.4075 \\
-0.0025 \\
109.6084 \\
67.1313 \\
-656.9457
\end{array}\right]
$$

Note that the parameters $\ddot{\theta}(75)$ are just one selection from the unfalsified set at $t_{k}=75 \mathrm{~s}$, and that the distance to the center of the unfalsified set differs per parameter. Furthermore, the resulting controller parameter set $\check{\theta}(75)$ is not in the initial ellipsoid $\mathcal{E}(0)$. This remarkable phenomenon is allowed by the outer-bounding approximations that let the ellipsoid extend in some directions.

\section{B. Frequency Domain}

The measured frequency response of the experimental setup (which is not used in the EUC algorithm), as derived

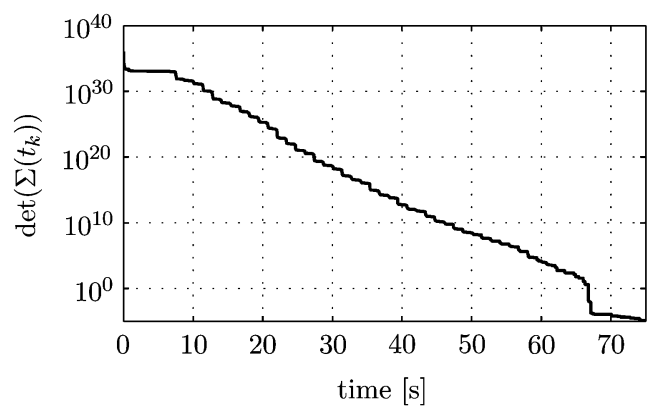

Fig. 9. Evolution of $\operatorname{det}\left(\Sigma\left(t_{k}\right)\right)$ over time, which is proportional to the volume of the unfalsified set $\mathcal{E}\left(t_{k}\right)$.

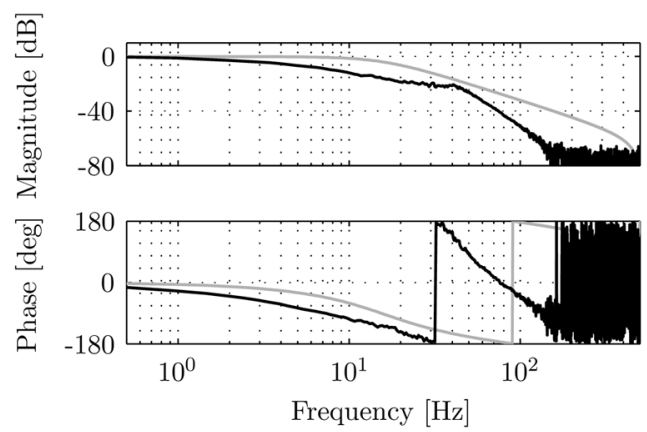

Fig. 10. Estimated frequency response of closed-loop system from $r$ to $y$ with control parameter set after $75 \mathrm{~s}$ (black) and frequency response of reference model $G_{m}(q)$ (grey). Only the linear filters of $w(\cdot)$ are regarded.

in Section III-A2, is used to construct the frequency response of the closed-loop system from $r$ to $y$ with the unfalsified controller after $75 \mathrm{~s}$. For this purpose, first only the linear filters of $w(\cdot),(23)$, are considered. The resulting frequency response is shown in Fig. 10. Most striking observation is the lower gain of the closed-loop frequency response compared to the frequency response of the reference model $G_{m}(q),(18)$. In Fig. 11, the frequency content is shown of the achieved output $y\left(t_{k}\right)$ and the desired output $G_{m}(q) r\left(t_{k}\right)$. The low-frequent deviation, as predicted by Fig. 10, is not observed. Actually, the frequency content matches up to $15 \mathrm{~Hz}$ in the peaks, where the SNR is largest. At frequencies higher then $15 \mathrm{~Hz}$, the achieved output $y\left(t_{k}\right)$ even contains more energy then $G_{m}(q) r\left(t_{k}\right)$. Therefore, an extension to the analysis as previously performed is proposed.

In the previous frequency response analysis, the influence of the nonlinear element of $w(\cdot),(23)$, is neglected. To incorporate the influence of this element, it is observed that the output signal of the nonlinear filter $\operatorname{sign}\left((1-q) r\left(t_{k}\right)\right)$ is exactly duplicated by the linear filter $\left(f_{s} / 2 f A\right)\left((1-q) r\left(t_{k}\right)\right)$ for the reference defined in (21) and zero initial conditions, with $f$ and $A$ as in (21) and $f_{s}$ the sample frequency $(1000 \mathrm{~Hz}$ for this setup). Since both filters produce exactly the same output signal for this specific $r\left(t_{k}\right)$, the control output is not changed if the nonlinear filter was replaced by the linear filter. For the frequency domain analyses, the linear filter can therefore be substituted for the nonlinear filter, and an analysis can be made of the frequency content of the output relative to the frequency content of the input of the closed-loop system incorporating the influence of the additional filter. This ratio of the frequency contents is shown in Fig. 12 


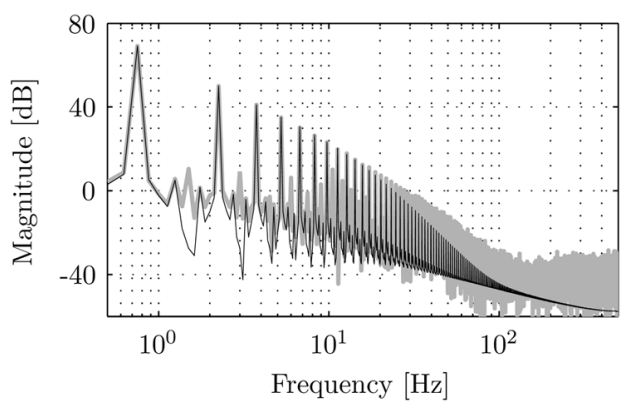

Fig. 11. Estimated frequency content of $G_{m}(q) r\left(t_{k}\right)$ (black) and $y\left(t_{k}\right)$ with $\check{\theta}(75)$ (grey).

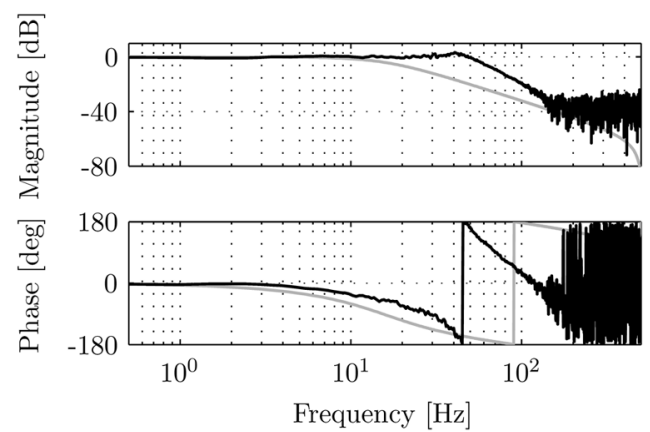

Fig. 12. Estimated frequency response of closed-loop system from $r$ to $y$ with control parameter set after $75 \mathrm{~s}$ (black) and frequency response of reference model $G_{m}(q)$ (grey), with substituted contribution of the nonlinear filter of $w(\cdot)$.

and the gain of the closed-loop transfer function increased drastically for frequencies larger then $1 \mathrm{~Hz}$ by incorporation of the filter.

The ratio of the frequency contents predicts good tracking for frequencies up to $12 \mathrm{~Hz}$, whereas the frequency response with the purely linear filters already showed a considerable deviation for frequencies larger then $2 \mathrm{~Hz}$. In the frequency contents, shown in Fig. 11, no large low frequent deviation is observed, therefore, it is concluded that the extended frequency response is more consistent with the observed behavior.

The analysis, as performed, before only holds for the specific triangular reference as is applied in the experiment. But then again, the resulting controller is specifically designed using the conditions as encountered in the experiment.

\section{Evaluation}

In the process of implementation of the proposed data-driven controller design strategy, several observations are made, which will be discussed here.

\section{A. Applicability of EUC}

Starting point for this research was to show the applicability of EUC to a real-life motion system, with as constraint a high sample frequency of $1 \mathrm{kHz}$. Even under the influence of the disturbances, as encountered in the experiment, this plant-model-free controller design method with continuous adaptation correctly adapted the unfalsified set and the implemented controller.

The little plant knowledge that was available from some preliminary experiments is used in the design of the controller structure and the performance requirement. No further plant knowledge is needed, since the controller parameters are adapted using measured input/output data. During the experiment, the controlled system satisfied the predefined performance requirement, except at the instances that the currently implemented controller was falsified and a new controller was inserted in the loop.

Because not much effort is needed in the design of the initial controller, only limited tuning effort is needed in the entire control design. Furthermore, this data-driven method is applied under normal operating conditions, no specially designed experiment is required. Accordingly, the method is very versatile and easily extendable.

\section{B. Remaining Issues in EUC}

Still, some open issues remain. Although theoretically a "plug-and-play" method, some aspects are less desirable or not yet fully understood.

First, the main aspect is the history dependency of the algorithm. The initial controller conditions and measurement data, as well as the parameter values for the algorithm, determine the switching sequence of the controller parameters. The switching of the controller parameters induces transient behavior, which results in falsification of control parameter sets that would have been unfalsified with other switching sequences. Consequently, the resulting unfalsified set is influenced by the initial control parameter set $\breve{\theta}(0)$ as well as by $\alpha$, the specific shape of $\Delta\left(t_{k}\right)$ and $\kappa\left(t_{k}\right)$ and the initial unfalsified set $\mathcal{E}(0)$. The measurement data that is generated on the system also depends on the specific controller sequence, which thereby influences the result. Ideally, the result (at least, the achievable performance) should be insensitive to initial choices and the accidental route of the implemented controller parameters.

Second aspect is the selection of the controller structure. It directly influences feasibility of the adaptive control system.

An "unfortunate" choice of the filters easily results in infeasibility or, at best, induces a very elongated ellipsoid, which leads to an ill-conditioned $\Sigma$, hence, an ill-conditioned update. This latter scenario happens for instance if the outputs of some filters are similar such that linear combinations of these filters result in the same time signal.

The third aspect arises from the desire to be able to establish a priori if the adaptive control problem is feasible. Currently, methods are lacking that could pass judgement on the chances of feasibility, if only some (limited) plant information is available. As a next step, a method could be derived that maximizes the chances of feasibility during the design of the controller structure.

As final aspect, the fictitious reference signal is used as a tool for the prediction of the performance of controllers, thereby eliminating the need to implement all controllers. However, since this fictitious signal differs from the real reference, the extrapolation of the performance to the actual reference might be inaccurate. Different dynamics might be emphasized or, on 
the contrary, not excited [3], resulting in the erroneous falsification or unfalsification of controllers. Consider for instance Fig. 1, where the transfer from $r$ to $r_{\text {fict }}\left(\theta^{\prime}\right)$ is given by

$$
r_{\text {fict }}\left(\theta^{\prime}\right)=\frac{C_{r}\left(\theta^{\prime}\right)^{-1} C_{r}(\check{\theta})\left(1+P C_{y}\left(\theta^{\prime}\right)\right)}{1+P C_{y}(\check{\theta})} r .
$$

Then, unstable poles of $(1) /\left(1+P C_{y}\left(\theta^{\prime}\right)\right)$ are not excited by $r_{\text {fict }}\left(\theta^{\prime}\right)$, because of the corresponding zeros in (27).

The extent of the deviation between $r$ and $r_{\text {fict }}$ is influenced by the (somewhat accidental) route of the implemented control parameter set, as already asserted in the first aspect.

\section{Possible Extensions}

Extensions are possible to the current theory of EUC that address some of the issues mentioned before. However, more research is needed, for instance, with respect to stability issues with these extensions.

To overcome the problem of the history dependency of the adaptive algorithm, modifications could be implemented that discard the history. Possibilities are to gradually increase $\Sigma$ as a kind of "forgetting factor," or to reset $\mathcal{E}$ to its initial volume and shape if the volume of the unfalsified set is below a certain threshold. However, this would destroy the stability proof if not done carefully, since the finite number of controller switches is no longer guaranteed with the current proof. Another option is batch-wise implementation of the algorithm, where the controller is only updated after a batch of data is acquired. However, this in turn induces other problems, e.g., if the implemented controller turns out to be destabilizing after all.

Based on, e.g., the shape of the ellipsoidal unfalsified set, the controller structure can be adapted. Non-essential filters can be discarded, linear combinations of filters can be merged and the structure can be extended with filters based on, e.g., the error signal. A supervisory loop can monitor the signals in the controller structure, and decide on these modifications. Attention should be payed to non-smooth transitions and initial conditions of the controller states. This extension, however, conflicts with the benefit of data-driven control design that the controller complexity is defined a priori, even supposed that the possible extensions of the controller structure are taken from a predefined filter library.

In the selection of the control parameters that are to be implemented additional constraints can be considered, such as, for instance, non-negativity constraints or the maximization/minimization of certain parameters. As examples, consider the selection of the maximal $\breve{\theta}_{1}$ to minimize control effort, or the selection of the minimal $\left|\breve{\theta}_{9}\right|$, to minimize the nonlinear contribution. Main point here is that the choice of parameters basically can be arbitrary, as long as it is from $\mathcal{E}\left(t_{k-1}\right) \cap \mathcal{U}\left(t_{k}\right)$. However, care should be taken with the guarantee of a finite number of switches and with possibly undesirably large steps in the controller parameters.

\section{CONCLUSION}

In this paper, the data-driven plant-model-free controller design method EUC is applied to a motion system.
Due to the specific design choices for the performance requirement and controller structure, in EUC the unfalsified set can be updated analytically. This results in a computationally cheap algorithm that can be implemented on a system with a sample frequency of $1 \mathrm{kHz}$ or higher.

The method is applied to an industrial inkjet printer at a sample rate of $1 \mathrm{kHz}$. The motivations of the design choices are presented and the experimental setup is discussed. The results clearly show that the algorithm updates the control parameter set when the performance requirement is not met with the currently implemented one. Meanwhile, the unfalsified set decreases almost continuously. The resulting closed-loop behavior resembles the predefined reference model in the dominant frequency range.

Even though the results are admirable, some issues still need to be addressed. The result of the algorithm is influenced both by its switching sequence and by the choice of the controller structure. Extension have been suggested using some sort of supervisory loop, such that the adaptive system can be reinitialized with different initial conditions or such that the controller structure is adapted.

\section{REFERENCES}

[1] S. Boyd, L. El Ghaoui, E. Feron, and V. Balakrishnan, Linear Matrix Inequalities in System and Control Theory. Philadelphia, PA: SIAM' studies in Applied Mathematics, 1994.

[2] F. B. Cabral and M. G. Safonov, "Unfalsified model reference adaptive control using the ellipsoid algorithm," Internal. J. Adapt. Control Signal Process., vol. 18, no. 8, pp. 683-696, Oct. 2004.

[3] S. Engell, T. Tometzki, and T. Wonghong, "A new approach to adaptive Unfalsified control," in Proc. Eur. Control Conf., Jul. 2007, pp. $1328-1333$.

[4] J. J. M. van Helvoort, "Unfalsified control: Data-driven control design for performance improvement," Ph.D. dissertation, Mech. Eng. Dept., Control Syst. Technol. Group, Technische Universiteit Eindhoven, Eindhoven, The Netherlands, Dec. 2007.

[5] J. J. M. van Helvoort, B. de Jager, and M. Steinbuch, "Data-driven multivariable controller design using ellipsoidal unfalsified control," in Proc. Amer. Control Conf., Jul. 2007, pp. 510-515.

[6] J. J. M. van Helvoort, B. de Jager, and M. Steinbuch, "Direct datadriven recursive controller unfalsification with analytic update," Automatica J. IFAC, vol. 43, no. 12, pp. 2034-2046, Dec. 2007.

[7] A. K. Katsaggelos, J. Biemond, R. W. Schafer, and R. M. Mersereau, "A regularized iterative image restoration algorithm," IEEE Trans. Signal Process., vol. 39, no. 4, pp. 914-929, Apr. 1991.

[8] D. G. Maksarov and J. P. Norton, "State bounding with ellipsoidal set description of the uncertainty," Internal. J. Control, vol. 65, no. 5, pp. 847-866, 1996.

[9] L. Pronzato and E. Walter, "Minimal volume ellipsoids," Internal. J. Adapt. Control Signal Process., vol. 8, pp. 15-30, 1994.

[10] L. Ros, A. Sabater, and F. Thomas, "An ellipsoidal calculus based on propagation and fusion," IEEE Trans. Syst. Man Cybern. B, Cybern., vol. 32, no. 4, pp. 430-442, Aug. 2002.

[11] M. G. Safonov and T.-C. Tsao, "The unfalsified control concept and learning," IEEE Trans. Autom. Control, vol. 42, no. 6, pp. 843-847, Jun. 1997.

[12] M. Stefanovic, A. Paul, and M. G. Safonov, "Safe adaptive switching through infinite controller set: Stability and convergence," presented at the IFAC World Congr., Prague, Czech Republic, Jul. 2005.

[13] T.-C. Tsao and M. G. Safonov, "Unfalsified direct adaptive control of a two-link robot arm," Internal. J. Adapt. Control Signal Process., vol. 15, no. 3, pp. 319-334, May 2001.

[14] B. R. Woodley, J. P. How, and R. L. Kosut, "Direct unfalsified controller design-Solution via convex optimization," in Proc. Amer. Control Conf., Jun. 1999, pp. 3302-3306. 


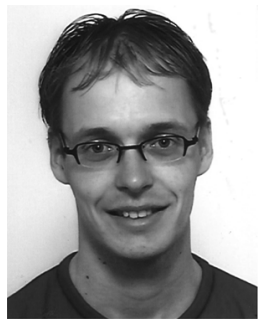

Jeroen van Helvoort was born in Vlijmen, The Netherlands, in 1980. He received the M.Sc. (cum laude) and Ph.D. degrees in mechanical engineering from the Technische Universiteit Eindhoven, Eindhoven, The Netherlands, in January 2004 and December 2007, respectively.

His research interests focus on data-driven and disturbance-based control with a special interest in unfalsified control.

Bram de Jager received the M.Sc. degree in mechanical engineering from Delft University of Technology, Delft, The Netherlands, and the Ph.D. degree from the Technische Universiteit Eindhoven, Eindhoven, The Netherlands.

Currently, he is with the Technische Universiteit Eindhoven. He was with Delft University of Technology and with Stork Boilers BV, Hengelo, The Netherlands. His research interests include robust control of (nonlinear) mechanical systems, integrated control and structural design for tensegrities, control of fluidic systems, control structure design, and application of symbolic computation in nonlinear control.

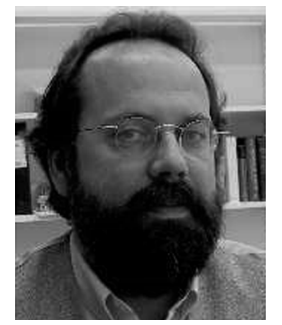

Maarten Steinbuch received the M.Sc. and Ph.D. degrees from Delft University of Technology, Delft, The Netherlands, in 1984 and 1989, respectively.

Since 1999, he has been a Full Professor with the Control Systems Technology Group, Mechanical Engineering Department, Eindhoven University of Technology, Eindhoven, The Netherlands. From 1987 to 1998 , he was with Philips Research Labs., Eindhoven, The Netherlands. From 1998 to 1999, he was a Manager of the Dynamics and Control Group, Philips Center for Manufacturing Technology. Since July 2006, he has also been a Scientific Director of the Centre of Competence High Tech Systems of the Federation of Dutch Technical Universities. His research interests include modeling, design, and control of motion systems and automotive powertrains.

Prof. Steinbuch was a recipient of the "Best-Teacher" Award of the Department of Mechanical Engineering, TU/e in 2003 and 2005. He was an Associate Editor of the IEEE Transactions on Control Systems Technology, of the IFAC Control Engineering Practice, and of the IEEE Control Systems Magazine. He is currently Editor-At-Large of the European Journal of Control. 